\title{
Terapia nutricional na hipertensão
}

\author{
Márcia R. S. T. Klein ${ }^{1^{*}}$
}

\section{Resumo}

A hipertensão arterial sistêmica (HAS) apresenta elevada prevalência e é considerada um dos principais fatores de risco para as doenças cardiovasculares, que representam a principal causa de morte no mundo. O risco cardiovascular aumenta de forma progressiva com a elevação da pressão arterial (PA) a partir de 115/75 mmHg. Inúmeros estudos avaliaram os efeitos sobre a PA de vários fatores nutricionais. Existem evidências consistentes de que a redução na ingestão de sódio dietético, a perda ponderal nos indivíduos com excesso de peso corporal, a moderação no consumo de álcool e o padrão alimentar da dieta DASH (Dietary Approaches to Stop Hypertension) podem reduzir a PA. Outros componentes da dieta provavelmente também interferem no controle da PA, porém seus efeitos são pequenos e/ou as evidências são inconclusivas. Nesta revisão, serão abordadas as evidências disponíveis na literatura sobre o papel de diferentes fatores dietéticos na prevenção e tratamento da HAS.

Descritores: Terapia nutricional; Dieta; Hipertensão; Pressão arterial.

\section{Abstract}

\section{Nutrition therapy in hypertension}

Arterial hypertension has a high prevalence and is considered one of the main risk factors for cardiovascular diseases, which are the leading cause of death worldwide. The risk of cardiovascular disease increases progressively with increasing blood pressure (BP) above 115/75 mmHg. Numerous studies have evaluated the effects on BP of several nutritional factors. There is consistent evidence that the reduction in dietary sodium intake, the weight loss in individuals with excess body weight, the moderation of alcohol consumption and the DASH (Dietary Approaches to Stop Hypertension) diet eating plan can lower BP. Other components of the diet probably also interfere with BP control, but its effects are small and/or the evidence is inconclusive. In this review, the available evidence in the literature on the role of different dietary factors in the prevention and treatment of hypertension will be addressed.

Keywords: Nutrition therapy; Diet; Hypertension; Blood pressure.
1. Departamento de Nutrição Aplicada. Instituto de Nutrição. Universidade do Estado do Rio de Janeiro. Rio de Janeiro, RJ, Brasil.

\section{"Endereço para correspondência:}

Boulevard 28 de setembro, 87, salas 361 e 363.

Rio de Janeiro, RJ, Brasil. CEP: 20551-030

E-mail:marciarsimas@gmail.com

Revista HUPE, Rio de Janeiro, 2015;14(3):34-40

doi: 10.12957/rhupe.2015.19893

Recebido em 19/03/2015. Aprovado em 03/08/2015.

\section{Resumen}

\section{Terapia nutricional en la hipertensión}

La hipertensión arterial tiene una alta prevalencia y es considerada uno de los principales factores de riesgo para enfermedades cardiovasculares, que son la principal causa de muerte en el mundo. El riesgo cardiovascular aumenta progresivamente a medida que aumenta la presión arterial (PA), comenzando a partir de 115/75 mmHg. Numerosos estudios han evaluado los efectos sobre la PA de varios factores nutricionales. Hay evidencia consistente de que la reducción en la ingesta de sodio en la dieta, la pérdida de peso en personas con exceso de peso corporal, la moderación en el consumo de alcohol y el patrón alimentario de la dieta DASH (Dietary Approaches to Stop Hypertension) puede reducir la PA. Otros componentes de la dieta probablemente también interfieren en el control de la PA, pero sus efectos son pequeños y/o la evidencia no es concluyente. En esta revisión, se abordará la evidencia disponible en la literatura sobre el papel de los diferentes factores de la dieta en la prevención y tratamiento de la hipertensión.

Palabras clave: Terapia nutricional; Dieta; Hipertensión; Presión arterial. 


\section{Introdução}

A hipertensão arterial sistêmica (HAS) é uma condição clínica multifatorial caracterizada por níveis elevados e sustentados de pressão arterial (PA), ${ }_{1}^{1}$ sendo definida por valores de PA sistólica > $140 \mathrm{mmHg}$ e/ou PA diastólica > 90 mmHg. ${ }^{1,2}$ A prevalência mundial de HAS é elevada, sendo de aproximadamente $40 \%$ nos adultos com idade $\geq 25$ anos. ${ }^{3}$ No Brasil, estudos indicam prevalência em torno de $30 \%$ na população adulta. ${ }^{1}$

A HAS apresenta baixas taxas de controle e frequentemente está associada com alterações estruturais e/ou funcionais de órgãos-alvo (ex.: coração, encéfalo, rins e vasos sanguíneos) e com alterações metabólicas, com consequente aumento no risco de doença renal crônica e de doenças cardiovasculares (DCV). 1,2 As DCV são a principal causa de morte em todo o mundo, sendo responsáveis por cerca de 30\% dos óbitos. A HAS é considerada um dos principais fatores de risco modificáveis para as DCV. O risco cardiovascular aumenta de forma progressiva e linear com a elevação da PA a partir de 115/75 mmHg. ${ }^{1}$ A Organização Mundial de Saúde (OMS) estima que em todo o mundo, a HAS cause cerca de $12,8 \%$ das mortes. ${ }^{3}$

Existem evidências de que a detecção, o tratamento e o controle da HAS são fundamentais para a redução dos eventos cardiovasculares. O tratamento da HAS pode ser subdividido em tratamento medicamentoso e tratamento não medicamentoso. ${ }^{1}$

O tratamento não medicamentoso inclui a intervenção nutricional e apresenta eficácia comprovada na prevenção e no controle da hipertensão arterial. ${ }^{4-6}$ As modificações no estilo de vida isoladamente podem ser suficientes para pacientes com PA levemente elevada e devem sempre ser recomendadas para pacientes recebendo drogas anti-hipertensivas, pois podem reduzir a dosagem dos anti-hipertensivos necessários para alcançar o controle da PA. ${ }^{7}$ As principais modificações no estilo de vida recomendadas na prevenção e tratamento da hipertensão incluem atividade física regular, cessação do tabagismo, perda ponderal nos indivíduos com excesso de peso corporal e modificações dietéticas. ${ }^{1,2,5-10}$ No presente artigo, serão abordadas as evidências disponíveis na literatura sobre o papel de diferentes fatores dietéticos na prevenção e tratamento da HAS.

\section{Sódio}

O sódio é um nutriente essencial, sendo necessário para a manutenção do volume de fluido extracelular, da osmolaridade sérica, do equilíbrio ácido-básico e da atividade muscular e nervosa. ${ }^{11,12}$ A quantidade mínima de sódio necessária para repor as perdas diárias, desde que não ocorra transpiração substancial, é muito pequena podendo ser menor que $0,2 \mathrm{~g} / \mathrm{dia}^{11}$

Como nenhum alimento em seu estado natural é rico em sódio, durante milhões de anos, os ancestrais dos seres humanos, consumiam uma dieta contendo pequena quantidade deste mineral ( $\pm 0,2$ g sódio/dia) ${ }^{13}$ Há cerca de 5.000 anos, foi descoberto que o sal poderia ser usado para a preservação dos alimentos, levando a um aumento acentuado no consumo de sódio. Posteriormente, a invenção da refrigeração e do congelamento dos alimentos, tornou desnecessária a adição de sal para preservação dos alimentos. Entretanto, o consumo de sódio permanece elevado em praticamente todas as populações. Atualmente, a ingestão de sódio em muitos países situa-se entre 3,6 e 4,9 g/dia (ou 9 a 12 g sal/dia). ${ }^{13,14}$

\section{Estudos epidemiológicos}

Estudos epidemiológicos realizados em diferentes regiões ao redor do mundo investigaram a relação entre ingestão de sódio e níveis de PA. Esta relação foi primeiramente explorada na publicação do famoso gráfico de Louis Dahl em 1960, que demonstrou uma relação linear positiva entre a prevalência de hipertensão e o consumo médio de sódio através de 5 populações. ${ }^{15}$ Em seguida, o estudo internacional sobre sal e pressão arterial, o INTERSALT (International Study of Salt and Blood Pressure) utilizou técnicas adequadas para mensurar a PA e a ingestão de sódio (excreção urinária de sódio de $24 \mathrm{~h}$ ) em uma grande amostra ( \pm 11.000 ) de homens e mulheres, com idade entre 20-59 anos, provenientes de 52 centros populacionais em 32 países localizados na Europa, Américas, Ásia e África. ${ }^{16}$ No INTERSALT, foi observada relação positiva e significativa da ingestão de sódio com os níveis de PA e com a prevalência de hipertensão. Essa associação permaneceu significativa mesmo após ajustes para variáveis que podem interferir nos níveis de PA como idade, sexo, peso corporal, ingestão de potássio e ingestão de álcool. Nas quatro populações primitivas que participaram do INTERSALT (incluindo os índios Yanomami e os índios do Xingu no Brasil) a ingestão de sódio era extremamente baixa, os níveis de PA também eram baixos e a PA aumentava muito pouco ou não aumentava com a idade. ${ }^{16}$

Evidências sobre a relação entre ingestão de sódio e PA também foram encontradas em estudos mais recentes. No estudo internacional sobre micronutrientes e pressão arterial INTERMAP (International Study on Micronutrient and Blood Pressure), foram avaliados 4.680 


\section{Artigo de revisão}

indivíduos com idade entre 40-59 anos provenientes de 17 centros populacionais e uma menor ingestão de sal resultou em níveis mais baixos de PA. Neste estudo, a ingestão de sódio foi estimada através de quatro recordatórios de 24 horas e de duas coletas de urina de 24 horas. ${ }^{17}$ Tanto no INTERSALT quanto no INTERMAP, a relação entre ingestão de sódio e PA foi avaliada em diferentes sociedades, envolvendo alguns centros populacionais com diferentes níveis de ingestão de sódio, o que pode ter favorecido o encontro de associação significativa entre sódio e PA. Por outro lado, o estudo EPIC-Norfolk (Norfolk Cohort of the European Prospective Investigation into Cancer) foi realizado somente na Europa, sendo incluídas apenas sociedades com consumo de sódio semelhante ao das sociedades industrializadas, havendo pequena diferença na ingestão de sódio entre os indivíduos. A ingestão de sódio variou de aproximadamente $2 \mathrm{~g}$ a $5 \mathrm{~g}$. Mesmo com esta pequena variação, foi possível observar que quanto maior a ingestão de sódio, mais elevada era a PA. ${ }^{18}$

\section{Estudos de intervenção}

Evidências consistentes, provenientes de vários estudos, indicam que a redução do sódio dietético reduz a PA tanto em hipertensos quanto em normotensos. As exceções são estudos nos quais: (1) as intervenções para reduzir o sódio dietético apresentaram pequeno efeito sobre a ingestão de sódio; (2) a duração foi muito curta; ou (3) o tamanho da amostra era pequeno. O maior efeito do tratamento foi observado em estudos com duração maior do que quatro semanas..$^{8,12}$

\section{Mecanismo de ação}

Apesar dos mecanismos responsáveis pela relação entre ingestão de sódio e elevação da PA serem complexos, a falta de habilidade renal para excretar completamente o excesso de sódio desempenha um papel central. ${ }^{12}$ Os rins humanos estão adaptados para conservar sódio e excretar potássio. Os humanos pré-históricos, que consumiam uma dieta pobre em sódio e rica em potássio, estavam bem adaptados a este mecanismo. Com tal dieta, a excreção de sódio é negligenciável e a excreção de potássio é alta, pareando a ingestão de potássio. Este mecanismo, entretanto, não está adequado para a dieta moderna rica em sódio e pobre em potássio. O resultado final é a falha dos rins em se adaptar a esta dieta com um excesso de sódio e déficit de potássio. ${ }^{19}$

O excesso de sódio tem um impacto nas células musculares lisas vasculares podendo aumentar a PA. ${ }^{12}$
A retenção de sódio inibe a bomba de sódio das células musculares lisas arteriais e arteriolares, aumentando a concentração intracelular de sódio, o que estimula o aumento da concentração intracelular de cálcio, desencadeando a contração do músculo liso vascular. A elevada ingestão de sódio também reduz a síntese de óxido nítrico, prejudicando a vasodilatação dependente do endotélio. ${ }^{19}$

\section{Sensibilidade ao sal}

A modificação da PA em resposta a mudanças na quantidade de ingestão de sódio varia amplamente entre os indivíduos. Esta variabilidade é conhecida como sensibilidade ao sal, sendo definida como a tendência para a PA cair durante a restrição de sal e se elevar durante a suplementação de sal. Os mecanismos envolvidos na sensibilidade ao sal ainda não são bem compreendidos. Os indivíduos sensíveis comumente têm um defeito na excreção renal de sódio que acarreta expansão do volume e culmina com hipertensão. A sensibilidade ao sal apresenta uma grande variedade de determinantes, incluindo fatores genéticos, etnia, idade, massa corporal e dieta (qualidade global da dieta, conteúdo de macro e micronutrientes), assim como a presença de doenças associadas (ex.: hipertensão, diabetes e disfunção renal).11,20

\section{Recomendação}

A OMS recomenda para a população em geral a redução na ingestão de sódio com o objetivo de reduzir a PA e o risco de DCV. ${ }^{8}$ A recomendação é ingestão < 2 g/dia de sódio ( 5 g/dia de sal) para adultos. Esta recomendação se aplica a indivíduos com e sem hipertensão e é semelhante a de inúmeras outras organizações e diretrizes para tratamento e prevenção de hipertensão e/ou DCV.1,5-7,9,10,21 Existem estimativas de que esta redução na ingestão de sódio possa reduzir a PA sistólica em aproximadamente 2 a $8 \mathrm{mmHg}$. ${ }^{1,921}$

Apenas aproximadamente 10\% do sódio dietético é proveniente dos alimentos naturais, enquanto o restante é proveniente do sal que é adicionado durante a cocção ou à mesa e proveniente de alimentos industrializados. Nos países de baixa renda onde as populações podem ter acesso limitado aos alimentos processados, o sal adicionado durante a cocção ou à mesa fornece a maior parte do sódio dietético. ${ }^{14}$ Nos países asiáticos, uma importante fonte de sódio é o molho de soja. Nos países desenvolvidos ou em desenvolvimento, o sal adicionado durante o processamento dos alimentos representa a maior parte do sódio dietético (75\% a 
80\%). ${ }^{10}$ Sendo assim, estratégias que visem uma redução substancial na ingestão de sal devem envolver esforços da indústria de alimentos e de restaurantes que devem reduzir gradativamente o sal adicionado aos alimentos. ${ }^{6}$ Em alguns países como o Japão, Inglaterra e a Finlândia, já foram adotadas estratégias populacionais visando reduzir a ingestão de sal, tendo sido alcançados resultados promissores na redução da PA. ${ }^{10}$

\section{Peso corporal}

O IMC está diretamente associado à elevação da PA e com a prevalência de hipertensão. O ganho ponderal, mesmo que modesto, aumenta de forma substancial o risco de hipertensão, enquanto que a perda ponderal reduz a $\mathrm{PA}{ }^{6}$

A prevalência de obesidade está aumentando de forma expressiva em todo o mundo, tanto em crianças quanto em adultos de diferentes grupos socioeconômicos, alcançando proporções epidêmicas. ${ }^{7}$ Dados da pesquisa nacional de saúde e exame nutricional, o National Health and Nutrition Examination Survey (NHANES), indicam que a prevalência de obesidade (índice de massa corporal [IMC] $>30 \mathrm{~kg} / \mathrm{m}^{2}$ ) nos Estados Unidos, em adultos com idade entre 20-74 anos, aumentou de 15\% em 1976-1980 para 35,7\% em 2009-2010. ${ }^{22}$ Tendências similares foram observadas na Europa, onde a prevalência de obesidade aumentou de forma acentuada nos últimos anos. ${ }^{23}$ No Brasil, segundo a Pesquisa de Orçamento Familiar de 2008-2009,24 o excesso de peso corporal (IMC $\geq 25 \mathrm{~kg} / \mathrm{m}^{2}$ ) foi detectado em cerca de metade dos homens $(50,1 \%)$ e das mulheres (48\%), com prevalência de 49\% entre os adultos de ambos os sexos. Já a obesidade (IMC $\geq 30 \mathrm{~kg} / \mathrm{m}^{2}$ ) foi observada em $14,8 \%$ do total de adultos (12,5\% dos homens e 16,9\% das mulheres).

Os mecanismos por meio dos quais a obesidade eleva a PA são complexos e incluem ativação do sistema renina angiotensina, aumento da atividade do sistema nervoso simpático e resistência à insulina. Além disto, a obesidade está associada ao aumento na reabsorção renal de sódio, alteração na pressão natriurese e expansão de volume. A obesidade também pode causar alterações estruturais acentuadas nos rins que eventualmente levam a doença renal crônica e posterior aumento da PA. Outros aspectos associados à obesidade podem favorecer a hipertensão como, por exemplo, a alteração na liberação de adipocinas (ex.: $\uparrow$ leptina e $\downarrow$ adiponectina), aumento na liberação de ácidos graxos livres, disfunção endotelial, inflamação sistêmica e apneia obstrutiva do sono. ${ }^{25}$

\section{Recomendação}

Os indivíduos hipertensos apresentando sobrepeso ou obesidade devem ser orientados a perder peso e idealmente alcançar IMC $<25 \mathrm{~kg} / \mathrm{m}^{2}$. 6,10,21 Entretanto, reduções na PA ocorrem antes e/ou sem alcançar o peso corporal desejado. Uma perda ponderal de apenas $5,1 \mathrm{~kg}$ pode reduzir a PA sistólica e a diastólica em 4,4 mmHg e 3,6 mmHg, respectivamente. ${ }^{26}$ A manutenção da circunferência da cintura $<102 \mathrm{~cm}$ em homens e $<88 \mathrm{~cm}$ em mulheres também é recomendada para a prevenção e tratamento da HAS.,10

\section{Potássio}

Alguns estudos sugerem que o potássio desempenha um importante papel no controle da PA. ${ }^{12,27}$ Dados epidemiológicos têm mostrado que populações com elevada ingestão de potássio apresentam valores mais baixos de PA e menor incidência de hipertensão arterial. ${ }^{20,27,28}$ Alguns estudos sugerem que uma dieta rica em potássio pode suprimir o aumento na PA causado pela elevação na ingestão de sódio e reduzir a sensibilidade ao sal. ${ }^{12,29} \mathrm{O}$ efeito hipotensor do potássio pode ser atribuído: (1) a natriurese induzida pela inibição da reabsorção de sódio nos túbulos proximais renais e a supressão da secreção de renina e (2) ao relaxamento do músculo liso vascular por aumento na produção de óxido nítrico, estimulo as bombas de sódio e abertura de canais de potássio. ${ }^{30}$

\section{Recomendação}

A OMS recomenda um aumento na ingestão de potássio para reduzir a PA e o risco de DCV, em adultos, sugerindo uma ingestão de pelo menos $90 \mathrm{mmol} /$ dia (3.510 mg/dia). ${ }^{27}$ O Programa de Educação em Hipertensão Canadense apresenta recomendação semelhante para indivíduos hipertensos: $>80 \mathrm{mmol} / \mathrm{dia} .{ }^{10}$ No Brasil, de acordo com os dados do Instituto Brasileiro de Geografia e Estatística (IBGE), a ingestão média deste mineral em indivíduos com idade $\geq 19$ anos é de aproximadamente $2.000-2.500 \mathrm{mg} / \mathrm{dia} .{ }^{24}$ As principais fontes dietéticas de potássio são frutas, vegetais, leguminosas e cerais integrais. Uma dieta rica em vegetais e frutas possibilita a ingestão de 2.000 a $4.000 \mathrm{mg}$ de potássio/dia. ${ }^{20}$ Deve-se ter o cuidado de não orientar este aumento na ingestão de potássio para pacientes com hipercalemia ou que estejam em risco de desenvolver esta condição. ${ }^{20}$ Não se recomenda o uso de suplementos de potássio como método de redução da PA. ${ }^{5}$ 


\section{Artigo de revisão}

\section{Cálcio}

Em estudos epidemiológicos, a ingestão de cálcio apresenta relação inversa com a PA. De forma similar, a suplementação de cálcio está associada à redução modesta da PA. ${ }^{31}$ VanMierlo e colaboradores ${ }^{32}$ realizaram uma meta-análise contendo 40 ensaios clínicos randomizados que avaliaram o efeito da suplementação de cálcio (dose média: $1.200 \mathrm{mg} /$ dia) e encontraram redução significativa da PA sistólica $(-1,86 \mathrm{mmHg})$ e diastólica $(-0,99 \mathrm{mmHg})$. A suplementação de cálcio apresenta maior efeito hipotensor em (1) indivíduos consumindo regularmente pequenas quantidades de cálcio; (2) indivíduos hipertensos ou em grupos com elevado risco de desenvolvimento de hipertensão, como indivíduos sensíveis ao sal e mulheres grávidas; e (3) estudos usando cálcio dietético ao invés do suplementar. ${ }^{31}$

O provável mecanismo de ação do cálcio sobre a PA envolve o fato de uma dieta pobre em cálcio aumentar a concentração intracelular de cálcio nas células do músculo liso vascular, resultando em vasoconstricção e consequente aumento da PA. ${ }^{31}$

\section{Recomendação}

Não há necessidade de se aumentar a ingestão de cálcio além do que é recomendado para a população em geral, tanto para prevenção como para o tratamento da hipertensão. De acordo com a academia nacional de medicina norte-americana, o Institute of Medicine (2010), ${ }^{33}$ a ingestão adequada de cálcio é de 1.000-1.200 mg/dia para todos indivíduos com idade $\geq 19$ anos, quantidade esta muito superior à ingestão habitual da população brasileira $( \pm 500 \mathrm{mg} / \mathrm{dia}) .{ }^{24}$ Como as principais fontes dietéticas de cálcio (70\%-75\%) são os laticínios, este nível de ingestão de cálcio pode ser alcançado através do consumo de 3 a 4 porções diárias de leite, queijo ou iogurte (de preferência os desnatados). O uso de suplementos de cálcio como método de redução da PA não deve ser recomendado. ${ }^{5,10}$

\section{Magnésio}

Ainda não existem evidências conclusivas sobre o efeito da suplementação de magnésio sobre a PA. Kass e colaboradores publicaram uma meta-análise envolvendo 22 estudos com suplementação de magnésio (120-973 mg/dia durante 3-24 semanas) e nem todos os estudos observaram redução da PA. Entretanto, foi observada redução significativa da PA sistólica (3-4 $\mathrm{mmHg}$ ) e da PA diastólica (2-3 mmHg). O efeito foi mais pronunciado nos estudos cruzados e nos estudos com maior dosagem de magnésio ( $>370 \mathrm{mg} / \mathrm{dia}){ }^{34}$

O mecanismo preciso pelo qual o magnésio pode auxiliar na redução da PA não é completamente conhecido. Entretanto, existem evidências de que o magnésio atua como um bloqueador natural de canal de cálcio, aumenta o óxido nítrico, melhora a função endotelial e induz a vasodilatação direta e indireta. ${ }^{35} \mathrm{~A}$ ingestão de magnésio recomendada para prevenção e tratamento de hipertensão deve ser igual à recomendada para a população em geral. O alcance desta quantidade deve ser realizado através de fontes alimentares, não sendo recomendado o uso de suplementos de magnésio como método de redução da PA..$^{5-10}$ De acordo com o Institute of Medicine (IOM), ${ }^{36}$ a ingestão adequada de magnésio para indivíduos com idade $\geq 19$ anos é de 310-320 mg/ dia para mulheres e de 400-420 para os homens, quantidades estas superiores a ingestão habitual da população brasileira ( $\pm 200-250 \mathrm{mg} /$ dia). ${ }^{24}$

\section{Álcool}

Oconsumo de quantidades excessivas deálcool está associado à elevação da $\mathrm{PA}^{2}$ e estima-se que seja responsável por 5\% a 7\% dos casos de hipertensão., ${ }^{4,6} \mathrm{~A}$ relação entre ingestão de álcool, níveis de PA e prevalência de hipertensão parece ser linear e existem evidências de que o consumo moderado pode não ser deletério em relação à elevação da PA. ${ }^{2}$ Desta forma, homens hipertensos que ingerem bebidas alcoólicas devem ser orientados a limitar seu consumo a não mais do que 20-30 g etanol/dia, já as mulheres hipertensas devem ingerir no máximo $10 \mathrm{~g}$ a $20 \mathrm{~g}$ etanol/dia. ${ }^{2}$ Para aqueles que não têm o hábito de ingerir bebidas alcoólicas, não se justifica recomendar que o façam. Estima-se que a moderação na ingestão de álcool pode reduzir a PA sistólica em 2-14 mmHg.

\section{Café}

O café é uma das bebidas mais populares em todo o mundo. O primeiro relato de que poderia aumentar a PA ocorreu há cerca de 80 anos. Acredita-se que a cafeína, um dos componentes do café, seja a responsável pela possível elevação da PA, pois apresenta efeito pressor agudo, principalmente em indivíduos hipertensos. Esse efeito pode ser mediado por uma série de mecanismos, incluindo a ativação do sistema nervoso simpático. ${ }^{37}$ Já foi demonstrado que, em indivíduos hipertensos, a ingestão de 200 mg a 300 mg de cafeína está associada à elevação aguda significativa da PA sistólica (em torno de $10 \mathrm{mmHg}$ ) e da PA diastólica (em torno de $5 \mathrm{mmHg}$ ). Este aumento na PA pode ser observado na primeira 
hora após a ingestão e persiste por até 3 horas. ${ }^{38}$

Apesar do café ser rico em cafeína e do seu consumo estar relacionado à elevação da PA em uma série de estudos, existem evidência de que seu efeito sobre a PA é bem menor do que seria esperado com base no seu conteúdo de cafeína..$^{39}$ Entretanto, em uma meta-análise publicada em 2012, envolvendo 10 ensaios clínicos randomizados e 5 estudos de coorte, o consumo do café não se associou à elevação significativa da PA nem ao maior risco de desenvolvimento de hipertensão. ${ }^{40}$

A possível explicação para o menor efeito pressor do café em comparação à cafeína é que o café possui outros componentes que apresentam efeito hipotensor (ex.: ácido clorogênico, flavonoides, magnésio e potássio) contrabalançando o efeito pressor da cafeína. Além disto, consumidores habituais de café desenvolvem tolerância ao efeito pressor da cafeína. ${ }^{37}$

Como os dados disponíveis atualmente são considerados insuficientes para se recomendar a favor ou contra o consumo de café em relação ao controle da PA, ${ }^{2}$ algumas diretrizes para tratamento da HAS desencorajam o consumo excessivo de café e de outros produtos ricos em cafeína ${ }^{5}$ ou informam que a elevação da PA causada pela cafeína, em doses habituais são irrelevantes. ${ }^{1}$

\section{Dieta Dash}

A dieta DASH (Dietary Approaches to Stop Hypertension) foi elaborada com o objetivo de investigar se um padrão alimentar "saudável" é capaz de reduzir os níveis de PA, independentemente de alguns fatores dietéticos que afetam a PA (ex.: ingestão de sódio, peso corporal e consumo de álcool). O padrão alimentar da dieta DASH é rico em frutas e hortaliças (4 a 5 porções/dia) e laticínios com baixo teor de gordura (2 a 3 porções/ dia), cereais integrais, frango, peixe e frutas oleaginosas; e pobre em doces, bebidas adoçadas e carne vermelha. Portanto, esta dieta é rica em potássio, magnésio, cálcio e fibras dietéticas, além de apresentar baixo teor de gordura (total e saturada) e colesterol. ${ }^{29}$ Já foi demonstrado que a dieta DASH é capaz de reduzir significativamente a PA, sendo recomendada a adoção desse padrão alimentar por indivíduos hipertensos e para a prevenção da hipertensão. ${ }^{1,2,10}$ Os benefícios sobre a PA têm sido atribuídos ao alto consumo de potássio, magnésio e cálcio. Estima-se que a adoção da dieta DASH pode reduzir a PA sistólica em 8-14 mmHg. ${ }^{1}$

\section{Considerações finais}

A intervenção nutricional apresenta um impor- tante papel tanto na prevenção quanto no tratamento da HAS. As principais modificações nutricionais que podem contribuir para a redução da PA incluem: redução na ingestão de sódio dietético, perda ponderal nos indivíduos com excesso de peso corporal, moderação no consumo de álcool e adoção do padrão alimentar da dieta DASH. Outros fatores dietéticos provavelmente podem afetar a PA, porém seus efeitos são pequenos e/ou as evidências são inconclusivas. Estudos futuros são necessários para elucidar o efeito desses e de novos fatores dietéticos sobre a PA, pois reduções, mesmo que pequenas, na PA podem reduzir o risco de DCV. A combinação de diferentes intervenções dietéticas pode ser mais efetiva do que a adoção de medidas isoladas.

\section{Referências}

1. Sociedade Brasileira de Cardiologia; Sociedade Brasileira de Hipertensão; Sociedade Brasileira de Nefrologia. VI Diretrizes brasileiras de hipertensão, 2010. Arq Bras Cardiol. 2010; 95(suppl.1):1-51.

2. European Society of Hypertension; European Society of Cardiology. Guidelines for the management of arterial hypertension. The task force for the management of arterial hypertension of the European Society of Hypertension (ESH) and European Society of Cardiology (ESC). J Hypertens. 2013; 31:1281-357.

3. World Health Organization. Global Health Observatory (GHO) data. Raised blood pressure. [Acesso em 2015 fev. 10]. Disponível em: http://www.who.int/gho/ncd/risk_factors/ blood_pressure_prevalence_text/en/\#

4. Raymond JL, Couch SC. Medical nutrition therapy for cardiovascular disease. In: Mahan LK, Escott-Stump S, Raymond JL, Krause MV. Krause's food \& the nutrition care process. 13 ed. St. Louis: Elsevier/Saunders, 2012. p.742-81.

5. National Institute for Health and Clinical Excellence. Clinical guideline 127 Hypertension: Clinical management of primary hypertension in adults, 2011. Londres (Reino Unido).

6. Appel LJ, Brands MW, Daniels SR, et al. Dietary approaches to prevent and treat hypertension. A scientific statement from the American Heart Association. Hypertension. 2006;47:296-308.

7. Perk J, Backer GD, Gohlke H, et al. European Guidelines on cardiovascular disease prevention in clinical practice (version 2012). Eur Heart J. 2012;33:635-70.

8. World Health Organization. Guideline: Sodium intake for adults and children. Geneva, 2012. [Acesso em 2015 fev. 10]. Disponível em: http://www.who.int/nutrition/publications/guidelines/sodium_intake/en/.

9. American Dietetic Association. Hypertension evidence based nutrition practice guideline. Chicago, 2008. [Acesso em 2014 set. 2]. Disponível em: http://www.guidelines.gov/content. aspx?id=12817\#Section420.

10. Hypertension Canada. Canadian Hypertension Education Program (CHEP) 2013 Recommendations. [Acesso em 2014 dez. 3]. Disponível em: http://www.hypertension.ca/chep.

11. Franco V, Oparil S. Salt sensitivity, a determinant of blood pressure, cardiovascular disease and survival. J Am Coll Nutr. 2006; 25(3):247S-55S.

12. Mohan S, Campbell NRC. Salt and high blood pressure. Clin Sci. 2009; 17(1):1-11. 
13. He FJ, Burnier M, MacGregor GA. Nutrition in cardiovascular disease: salt in hypertension and heart failure. Eur Heart J. 2011;32(24):3073-80.

14. Campbell NRC, Johnson JA, Campbell TS. Sodium Consumption: An Individual's Choice? Int J Hypertens. 2012; 2012: 860954.

15. Brown IJ, Tzoulaki IT, Candeias V, et al. Salt intakes around the world: implications for public health. Int J Epidemiol. 2009;38(3):791-813.

16. Intersalt Cooperative Research Group. INTERSALT: an international study of electrolyte excretion and blood pressure: results for $24 \mathrm{~h}$ urinary sodium and potassium excretion. BMJ. 1998; 297(6674):319-28.

17. Stamler J, Elliott P, Dennis B, et al. INTERMAP: background, aims, design, methods, and descriptive statistics (nondietary). J Hum Hypertens. 2003;17(9):591-608.

18. Khaw KT, Bingham S, Welch A, et al. Blood pressure and urinary sodium in men and women: the Norfolk Cohort of the European Prospective Investigation into Cancer (EPIC-Norfolk). Am J Clin Nutr. 2004;80(5):1397-403.

19. Adrogue HJ, Madias NE. Sodium and Potassium in the pathogenesis of Hypertension. N Engl J Med. 2007; 356:1966-78.

20. Roriz Filho JS, Nobre F, Coelho EB, et al. Tratmento não medicamentoso: sódio, potássio, cálcio e magnésio. In: Brandão AA, Amodeo C, Nobre F. Hipertensão. 2.ed. Rio de Janeiro: Elsevier. 2012;1(2):211-6.

21. Chobanian AV, Bakris GL, Black HR, et al. Seventh report of the Joint National Committee on Prevention, Detection, Evaluation, and Treatment of High Blood Pressure. Hypertension. 2003;42(6):1206-52.

22. Centers for Disease Control and Prevention. [Acesso em 2014 dez. 3]. Disponível em: http://www.cdc.gov/obesity/adult/index.html.

23. Tsigos C, Hainer V, Basdevant A, et al. Management of obesity in adults: European clinical practice guidelines. Obes Facts 2008; 1(2):106-16.

24. Instituto Brasileiro de Geografia e Estatística Pesquisa de Orçamentos Familiares 2008-2009: análise da disponibilidade domiciliar de alimentos e do estado nutricional no Brasil. Rio de Janeiro, 2010.

25. Kurukulasuriya LR, Stars S, Lastra G, et al. Hypertension in Obesity. Med Clin N Am. 2011;95(5):903-17.

26. Neter JE, Stam BE, Kok FJ, et al. Influence of weight reduction on blood pressure: a meta-analysis of randomized controlled trials. Hypertension. 2003;42(5):878-84.

27. World Health Organization. Guideline: Potassium intake for adults and children. Geneva, 2012. [Acesso em 2015 jan. 21]. Disponível em: http://www.who.int/nutrition/publications/guide- lines/potassium_intake/en/.

28. Kieneker LM, Gansevoort RT, Mukamal KJ, et al. Urinary potassium excretion and risk of developing hypertension The Prevention of Renal and Vascular End-Stage Disease Study. Hypertension. 2014;64:769-76.

29. Rodrigues SL, Baldo MP, Machado RC, et al. High potassium intake blunts the effect of elevated sodium intake on blood pressure levels. J Am Soc Hypertens 2014;8(4):232-8.

30. Nguyen H, Odelola OA, Rangaswami J, et al. A Review of Nutritional Factors in Hypertension Management. Int J Hypertens. 2013;2013:698940. doi:10.1155/2013/698940.

31. Torres MRSG, Sanjuliani AF. Does calcium intake affect cardiovascular risk factors and/or events? Clinics. 2012; 67(7):839-44.

32. vanMierlo LA, Arends LR, Streppel MT, et al. Blood pressure response to calcium supplementation: a metaanalysis of randomized controlled trials. J Hum Hypertens. 2006; 20(8):571-80.

33. Institute of Medicine. Dietary reference intakes for calcium and vitamin D. Report Breef. 2010. Disponível em: http://www. iom.edu/Reports/2010/Dietary-Reference-Intakes-for-Calcium-and-Vitamin-D.aspx

34. Kass L, Weekes J, Carpenter L. Effect of magnesium supplementation on blood pressure: a meta-analysis. Eur J Clin Nutr. 2012 Apr;66(4):411-8.

35. Houston M. The Role of Magnesium in Hypertension and Cardiovascular Disease. J Clin Hypertens (Greenwich). 2011;13:843-7.

36. Institute of Medicine. Dietary reference intakes for calcium, phosphorus, magnesium, vitamin D, and fluoride. Washington (DC): National Academies Press (US); 1997. Disponível em: http://www.ncbi.nlm.nih.gov/books/NBK109825/

37. Zhang Z, Hu G, Caballero B, et al. Habitual coffee consumption and risk of hypertension: a systematic review and meta-analysis of prospective observational studies. Am J Clin Nutr. 2011;93(6):1212-9.

38. Mesas AE, Leon-Muñoz LM, Rodriguez-Artalejo F, et al. The effect of coffee on blood pressure and cardiovascular disease in hypertensive individuals: a systematic review and meta-analysis. Am J Clin Nutr. 2011;94(4):1113-26.

39. Noordzij M, Uiterwaal CS, Arends LR, et al. Blood pressure response to chronic intake of coffee and caffeine: a meta-analysis of randomized controlled trials. J Hypertens. 2005 May;23(5):921-8.

40. Steffen M, Kuhle C, Hensrud D, et al. The effect of coffee consumption on blood pressure and the development of hypertension: a systematic review and meta-analysis. J Hypertens. 2012;30:2245-54. 